\title{
The interrelation of post-trauma stress disorders with reactive and personal anxiety
}

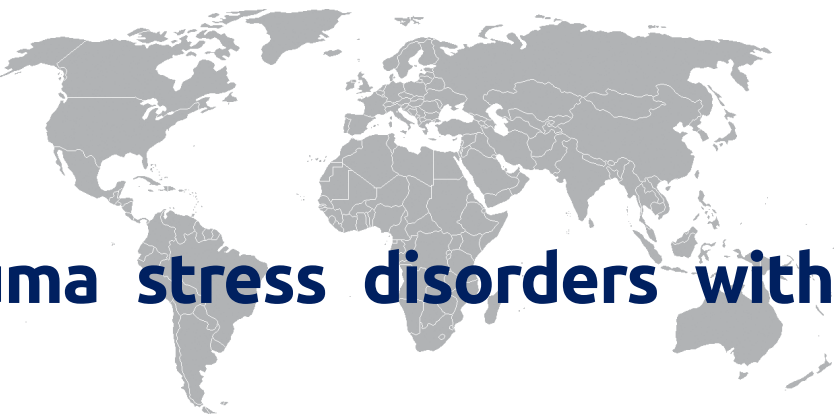

\author{
Irina V. Lopatkova1, Anna B. Serykh², Dmitriy V. Miroshkin ${ }^{3}$, Olga I. Shcherbakova ${ }^{4}$ Igor G. Kochetkov ${ }^{5}$
} Nelya A. Deberdeeva ${ }^{4}$, Ekaterina V. Diatlova ${ }^{3}$

\begin{abstract}
Objective: The relationship of situational (reactive) and personal anxiety in the sample of respondents with post-trauma stress disorder (PTSD) has been considered in the article. The study found that a significant level of situational anxiety and an average level of personal anxiety is observed in respondents with post-trauma stress disorder.

Method: The survey and testing of respondents was conducted on the basis of their voluntary and anonymous participation among the staff of public authorities and law enforcement bodies. Clinical, psychopathological and statistical research methods were used in this work. While conducting a psychopathological survey, the Mississippi scale (MS), military and civilian variants, the severity questionnaire for psychopathological symptoms (AFPS), and the scale for assessing the level of reactive and personal anxiety (Charles D. Spilberger, Yu. L. Khanin) were used to determine the qualitative and quantitative parameters. The levels of reactive and personal anxiety were determined on a scale: up to 30 points - low anxiety; from 31 to 45 points moderate anxiety; 46 points or more - high anxiety. The statistical processing of research results included the calculation of the arithmetic mean value for the quantitative (M) and qualitative $(P)$ indices and their errors ( $m$ and $p$ ). When comparing the data obtained in groups and subgroups, the Student's t-test was used, while the correlation analysis of interrelationships - the Spearman correlation coefficient was used. The sample comprised respondents with signs that meet the diagnostic criteria for PTSD (ICD-10 \DSM-R).

Results: The manifestation of the influence of the experienced psychological trauma on the level of anxiety is: an increased heart rate; Blanchet syndrome; a nervous condition; tension of all groups of muscles; feeling a sense of guilt; a feeling of fever followed by shivers; long confusion; insomnia; migraine; uncontrollable anger. The symptoms of anxiety persist and accumulate, at the time of a stressful situation, and after its resolution, contributing to a decrease in performance, fatigue and exhaustion. In addition, the results of the study indicate that anxiety and its level plays an important role, both in clinical findings and in the pathogenesis of PTSD. At the same time, differences were found among men and women. Men with PTSD are characterized by an increase in the level of situational anxiety as the severity of the disorder increases. Women are characterized by a moderate level of situational anxiety, regardless of the severity of the disorder. For both male and female subjects, high indices of personal anxiety are more significant than situational in the development of PTSD of different severity levels.

Conclusion: Anxiety and its level, plays an important role both in clinical findings and in the pathogenesis of PTSD. At the same time, differences were found among men and women. Men with PTSD are characterized by an increase in the level of situational anxiety as the severity of the disorder increases. Women are characterized by a moderate level of situational anxiety, regardless of the severity of the disorder. High indices of personal anxiety are more significant for both male and female subjects than situational one in the development of PTSD of different severity levels. The level of personal anxiety in men increased as the severity of PTSD increased. In women, the high level of anxiety increased with increasing severity of the disorder and stabilized with severe PTSD. Most women and men with PTSD are characterized by a high level of personal anxiety. Moreover, the prevalence of women with a high level of personal anxiety in comparison with men is essential with a mild severity of the disorder. With a moderate degree of the disorder, the differences in the proportion of men and women with a high level of personal anxiety are practically insignificant, and in case of a severe degree of the disorder, their proportion levels off, reaching $100 \%$, both among men and women.
\end{abstract}

Keywords: post-trauma stress disorder (PTSD), personal anxiety, situational (reactive) anxiety, "combat neurosis"

\footnotetext{
1 Moscow State University of Education, Moscow, Russia.

2 Institute of Humanities of Kant Baltic Federal University, Kaliningrad, Russia.

3 Sechenov First Moscow State Medical University, Moscow, Russia.

4 Plekhanov Russian University of Economics, Moscow, Russia.

5 Ulyanovsk State University, Ulyanovsk, Russia.
}

Correspondence: Igor G. Kochetkov

Ulyanovsk State University, Ulyanovsk, Russia.

E-mail: ikochetkov@yandex.ru

Received: 3 May 2018, Accepted: 30 Sep 2018

(C) 2018 by the authors; licensee Modestum Ltd., UK. This article is an open access article distributed under the terms and conditions of the Creative Commons Attribution License (http://creativecommons.org/licenses/by/4.0/).

Electronic Journal of General Medicine 


\section{INTRODUCTION}

The growth of the number of mentally traumatized people is now becoming increasingly common. In recent decades, the study of long-term effects of severe stress suffered by an individual, including post-trauma stress disorder (PTSD), is of great scientific interest.

According to numerous observations of specialists, the real clinical picture of PTSD can be much intense and more complicated than it is described in ICD-10 (heading F43.1) $(3,9,20,24,34,35)$. It is shown that PTSD is characterized by a polymorphic clinical picture (9). Anxiety is not only one of the obligate manifestations of PTSD (9), but also an important link in the mechanism of development of the disorder (19) an anxious type of PTSD has been identified and described in the literature (39). At the same time, some authors tend to view anxiety as a comorbid pathology in case of PTSD, occurring approximately equally among both men and women (5).

Today there is no consensus about the features of disorders connected with anxiety among men and women under stress. A number of authors believe that, against the background of stress, anxiety manifestations are detected more often in women than in men $(1,25)$. It is believed that this may be partly due to the neurophysiological characteristics of women. Thus, the interrelation of low activity of one of the enzymes regulating catecholamine neurotransmitter systems - COMT (catechol-O-methyltransferase) with instability under stress conditions has been described, which manifests itself in an increased frequency of anxiety disorders among women (12). At the same time, the authors note that this pattern is not found in men.

According to other researchers, anxiety after a trauma is a significant predictor of the development of PTSD in men, but not in women (1). The results of a study of levels of reactive and personal anxiety with PTSD of different severity levels have been presented in the domestic literature (25). The author believes that in the clinical picture and in the mechanism of development of PTSD, the level of personal anxiety is most significant, which increases with the rise of the disorder severity, and the level of situational anxiety remains almost unchanged. At the same time, a purpose-oriented study of situational and personal anxiety in PTSD of different severity levels in men and women was not conducted, which led to the research, the results of which are presented in this article.

\section{MATERIALS AND METHODS}

Post-trauma stress disorder (PTSD), according to epidemiological studies conducted by foreign authors, is detected among the population in 8-9\% of cases and is the second most common stress disorder in the United States (21). It is emphasized that the disorder develops in $25 \%$ of individuals exposed to an extreme stressful situation. Purpose-oriented epidemiological studies of the prevalence of PTSD in our country have not been conducted. One of the main criteria for the diagnosis of PTSD for ICD-10 is the etiological factor - the impact of a severe stressor. In this regard, the disorder is referred to psychogenic ones.

The incidence rates of PTSD differ depending on the type of injury, however, only some of the people exposed to the same traumatic factor develop PTSD (11). Many stressors, in response to the effects of which PTSD develops, are associated with the death of people. The severity and scale of military operations, technological disasters, terrorist acts, natural disasters, natural disasters and other emergency situations are determined by the number of human victims and the massive destruction of buildings and facilities that provide the living activity of people. Such events pose a threat to the life and health of people, some of whom die, while the others experience physical and mental suffering, and are referred to severe stressors.

The population groups are described in which PTSD can be developed as a sequence of exposure to severe stressors $(16,28)$. The authors indicate that one group is represented by subjects who were directly exposed to a traumatic stressor of high-intensity. The second group of the population includes those who were in close proximity to the accident while being on duty (accidentally) or experienced indirect effects of a stressor: rescuers, relatives of the dead or injured (16, 28), medical personnel (16), firefighters (37). The third group consists of those who have experienced stress, as a rule, of an informational nature, associated with the mass death of people under the influence of a strong stressor (28). All these people have a high risk of developing PTSD. It is now recognized that every suffering person in these groups deserves medical and psychological attention and help.

Military operations are the largest in scale and potentially life threatening to people. The consequences of the combat stress influence, including the development of PTSD, have been studied most often for more than 100 years and are still being studied now $(2,16,26,27,28,30,31,32)$. The modern warfare is connected not so much with the capture of 
territories, as it is aimed at killing people and inflicting physical harm to them - taking hostages, acts of terrorism. The development of PTSD in hostages, in victims of terrorist acts $(16,21,29)$ has been described.

Natural disasters, man-made disasters are also accompanied by a threat to life and health and mass death of people. PTSD is diagnosed in many victims (16). The development of PTSD is described in victims of car accidents and other railroad accidents, among migrants and IDPs; refugees, cancer patients $(16,28)$. A common factor among such stressors is also a high risk of dying or a damage to health, which leads to the formation of stress in people. The loss of close relatives is a particular mental trauma and can lead to the development of $\operatorname{PTSD}(6,7,22,27,33)$.

The experience gained over the past decades in domestic psychiatry, allows specialists to diagnose PTSD much more widely than at the initial stages of its study. However, the diagnosis of PTSD in the death of close relatives so far in practice has rarely been made which is groundless. The fact that the death of emotionally significant people (relatives, friends, colleagues, etc.) accompanies almost every person in life leads to the fact that experiences associated with this type of mental trauma sometimes remain without proper attention from specialists, and patients do not get proper medical care.

At present, there are no clear criteria to determine the stressful event contributing to the development of PTSD, which would be unequivocally interpreted (16). It is presented in ICD-10 that in order to diagnose PTSD, a person should be exposed to a stressful event or situation (both short and long) of an exclusively threatening or catastrophic nature, capable of causing general distress in almost any person. Such a definition does not give a clear idea of the nature of the stressor, which can lead to the development of PTSD, as well as to identify risk factors (16).

It seems that this may be one of the causes of the hyperdiagnosis of PTSD, especially in those who survived a mass destruction and death. On the other hand, an ambiguous definition of the severity of certain stressors and attitudes towards them (particularly when experiencing mentally traumatic events that are usually individual in nature: physical violence, especially in the family, death of close relatives, loss of work or housing, forced migration) leads to the fact that the disorder is not diagnosed (16). The fact that a stressor can be both a cause and a risk factor for the development of other psychogenic disorders, and not just PTSD contributes to it.

Determining the criteria for injury severity would allow one to structure stressors more clearly. A vital threat and personal psychological suffering caused by the death and suffering of other people (identification) can be considered as one of these signs. From this point of view, war, man-made disasters, transport, natural (fire) or natural (flood, tsunami) cataclysms, various kinds of violence (physical, sexual, psychological) have a common characteristic - a threat to people's lives and / or damage to their personal structures.

At the same time, PTSD is not developed in every person, even in those who were directly under the conditions of a high-intensity stressor. In this regard, we study the risk factors for the development of PTSD, among which external and internal are distinguished: etiological, biological, socio-psychological, personal, and others $(15,33)$.

The major clusters of factors that have influence on the formation and development of PTSD are identified:

1) factors that are directly related to an extreme influence;

2) psychological characteristics of the personality;

3) psychosocial influence of the nearest milieu;

4) mental injury in childhood;

5) a chronic mental injury;

6) factors connected with the attitude of the state and society (23).

A wide range of risk factors of developing PTSD are currently being studied. It is indicated that the nature of the stressor affects the possibility of developing PTSD: strength, a sudden character of action, duration of time (acute, chronic), direct or potential, type, content, intensity (28). The results of the study of the PTSD development in case of the loss of: a spouse, children and parents (22). It has been shown that the death of relatives and friends is the cause of developing PTSD, and the severity of the disorder depends on several factors, including the cause of death (22), for example, cancer disease, violent death, accident, murder, suicide (41). Gender differences of post-traumatic cognitive and emotional experiences of the death of the child by the mother and father are identified (22). A link was found between the development of PTSD and the degree of kinship with a deceased person (28).

In addition to external factors, the important role of internal risk factors for the PTSD development is discussed in the literature: biological validity, personality characteristics, its orientation, gender, age, life experience, resistance to stress, 
the presence of previous mental, psychosomatic disorders, somatic diseases, brain injuries, wounds, addictions (23). A number of authors consider the identification of PTSD markers promising, including biological (11), and biological indicators of emotional disorders in traumatized individuals (23). The age of an injured person is referred to the risk factors of the PTSD development, including in death of close relatives (37). An important role in the development of PTSD is given to the personality characteristics of a person, including the loss of people of extraordinary significance, among which is an individual predisposition to experiencing stress (2). Special attention is paid to the attitude of a traumatized person to a mentally traumatic event (31).

The opinion is expressed that the occurrence of psychogeny is often caused not by the person's immediate reaction to mental trauma, but by his ability to rework psychic trauma and the inability to adapt to the new conditions created (36), which is directly related to the loss of a close relative. The development of PTSD is associated with susceptibility to stress. Stress resistance is considered as a personality characteristic, including strength, psychological flexibility, creativity and the ability to restore normal functioning after severe stress, the presence of personal skills that help overcome trauma, and is determined by extrovert personality traits, such as endurance, superego stability, self-esteem, confidence and ability to mobilize internal resources (2).

Personality immaturity, asthenic traits, dependence, hypersensitivity, propensity for excessive control aimed at suppressing unwanted emotions, as well as trauma features - retention of traumatic experience and propensity for victimization (tendency to be in the role of a victim in similar situations) refer to pathogenic personality traits that determine an individual's vulnerability to PTSD (38). People who are highly sensitive to stress are considered to be more susceptible to traumatic events, and they show more pronounced signs of PTSD $(8,15)$. At the same time, according to some authors, the number of traumatic events have a relatively less effect on the development of PTSD among people with low sensitivity to stress.

Individual sensitivity to the psycho-traumatic situation of the loss of a significant relative may be related to the level of socio-psychological development of a person, which can be determined by the type of education. It is believed that PTSD develops much more often in those people whose childhood passed in problem families, and who experienced violence, psychological pressure and humiliation and cruel treatment in childhood (23). This subsequently leads to a decrease in resistance, which increases the risk of developing PTSD in the adult period of life.

Thus, at present, the study of risk factors of the PTSD development continues and is considered in the totality of the interdependence of biological, psychological and social aspects (13). In domestic psychiatry, a tendency emerged to study the causes of the development of affective disorders in mental disorders, including those caused by stress, as a result of the interaction between predispositional characteristics (personality traits, temperament, cognitive schemes / styles) and environmental factors (40). This interaction can be mediated by additional variables - social support, coping styles, personal resources, whose role is considered to be as protective or buffer one.

The analysis of the literature data testifies to a prospect and need to continue studying the etiology and risk factors of the PTSD development.

An overwhelming number of contemporary authors identify three main groups of PTSD symptoms:

1) hysterical response and its features (nervous tremor, blindness, deafness, paralysis);

2) excessive excitement (anxiety, sleep disturbance, vegetative lability, intrusive memories, avoidance of stressful situations,

recalling a traumatic situation);

3) periodic bouts of depressive mood (despair, emotional stupor, hopelessness) (17).

Additionally, neurotic syndromes corresponding to conditions in PTSD are identified:

1) "soldier's heart" (chest pain, sweating);

2) "survival syndrome" (a chronic sense of guilt);

3) "flashback syndrome" (memories of an experienced situation that suddenly appear in consciousness).

These symptom complexes, such as the flashback syndrome, are not stable, and, therefore, can both intensify and weaken over time (4). 
The publications of domestic authors present the results of studies of the levels of reactive and personal anxiety in PTSD of different severity levels (9). However, no detailed studies have been conducted at present, with a focus on the interdependence between PTSD and the level of personal and reactive anxiety.

The article presents the results of the study of the interdependence between PTSD and the level of situational and personal anxiety over time. A total number of 38 people aged from 33 to 57 were examined, of whom 27 were men (71\%) and 11 women (29\%). The average age of men was $43.8+0.85$ years, women $-44.9+0.9$ years.

The survey and testing of respondents was conducted on the basis of their voluntary and anonymous participation among the staff of public authorities and law enforcement bodies. Clinical, psychopathological and statistical research methods were used in this work. While conducting a psychopathological survey, the Mississippi scale (MS), military and civilian variants, the severity questionnaire for psychopathological symptoms (AFPS), and the scale for assessing the level of reactive and personal anxiety (Charles D. Spilberger, Yu. L. Khanin) were used to determine the qualitative and quantitative parameters. The levels of reactive and personal anxiety were determined on a scale: up to 30 points - low anxiety; from 31 to 45 points - moderate anxiety; 46 points or more - high anxiety.

The statistical processing of research results included the calculation of the arithmetic mean value for the quantitative $(\mathrm{M})$ and qualitative $(\mathrm{P})$ indices and their errors ( $\mathrm{m}$ and $\mathrm{p}$ ). When comparing the data obtained in groups and subgroups, the Student's t-test was used, while the correlation analysis of interrelationships - the Spearman correlation coefficient was used.

The sample comprised respondents with signs that meet the diagnostic criteria for PTSD (ICD-10 \DSM-R).

The required conditions were:

1) a history of mental trauma, acute or chronic one in its course;

2) clinical signs of PTSD;

3) several years have passed after the influence of a trauma.

Additionally, the results of a psychopathological study on the Mississippi traumatic disorder scale should correspond in subjects at least to 70 points, which indicates that they have PTSD (37).

\section{RESULTS AND DISCUSSION}

While conducting a Spearman correlation analysis between PSTD and the level of situational anxiety, a positive correlation coefficient was found to be 0.79 ; between PTSD and the level of personal anxiety, a positive correlation coefficient was found equal to 0.53 (with a significance level at $p<0.05$, where the sample size is $n=38$ and the critical value is $p^{\prime}=0.320$ ).

The study revealed that the level of situational anxiety among male respondents is $12.5+0.56$, while the level of personal anxiety is $10.5+0.36$.

The same tendency is observed for women respondents whose situational anxiety is $21.72+0.73$, in contrast to the level of personal anxiety, which is $17.72+0.7$.

The obtained findings suggest an increased level of anxiety in all surveyed respondents, with prevalence of anxiety in surveyed women and a more significant level of situational anxiety for respondents, characterized by severe anxiety, a feeling of emptiness, fast heart beat, migraine, hopelessness and outbursts of uncontrolled anger.

The analysis of the data obtained allowed us to identify a high correlation relationship between PTSD and situational anxiety in respondents, as well as an average relationship with personal anxiety.

The manifestation of the influence of the psychotrauma experienced on the level of anxiety is: an increased heart rate; Blanchet syndrome; a nervous condition; tension of all groups of muscles; feeling a sense of guilt; feeling a fever followed by shivers; long confusion; insomnia; migraine; uncontrollable anger. Symptoms of anxiety persist and accumulate during a stressful situation, and after its resolution, contributing to a decrease in performance, fatigue and exhaustion (10, 14, 18).

\section{CONCLUSION}

Thus, anxiety and its level, plays an important role both in clinical findings and in the pathogenesis of PTSD. At the same time, differences were found among men and women. Men with PTSD are characterized by an increase in the level of situational anxiety as the severity of the disorder increases. Women are characterized by a moderate level of situational anxiety, regardless of the severity of the disorder. 
High indices of personal anxiety are more significant for both male and female subjects than situational one in the development of PTSD of different severity levels. The level of personal anxiety in men increased as the severity of PTSD increased. In women, the high level of anxiety increased with increasing severity of the disorder and stabilized with severe PTSD. Most women and men with PTSD are characterized by a high level of personal anxiety. Moreover, the prevalence of women with a high level of personal anxiety in comparison with men is essential with a mild severity of the disorder. With a moderate degree of the disorder, the differences in the proportion of men and women with a high level of personal anxiety are practically insignificant, and in case of a severe degree of the disorder, their proportion levels off, reaching $100 \%$, both among men and women.

\section{REFERENCES}

1. Afifi M. Gender differences in mental health. Singapore Med. J. 2007;48(5):385-391. PMid:17453094

2. Agaibi CE, Wilson JP. Trauma PTSD and Resilience: A Review of the Literature. Trauma Violence Abuse. 2005;6(3):195-121. https://doi.org/10.1177/1524838005277438 PMid:16237155

3. Aleksandrovsky YuA. Border mental disorders: a guide for doctors. Moscow: GEOTAR-Media; 2007.

4. Andryushchenko AV. Post-traumatic stress disorder in situations of loss of object of extraordinary importance. Psychiatry and psychopharmacotherapy. 2000;4:104-109.

5. Avedisova AS. Psychopharmacotherapy of patients with post-traumatic stress disorder. Journal of Neurology and Psychiatry. 2009;109(12):46-49.

6. Boelen P. Intolerance of uncertainty and emotional distress following the death of a loved one. Anxiety Stress Coping. 2010;23(4):471-478. https://doi.org/10.1080/10615800903494135 PMid:20455126

7. Boelen PA, Huntjens RJ, van Deursen DS, van den Hout MA. Autobiographical memory specificity and symptoms of complicated grief, depression, and posttraumatic stress disorder following loss. Journal Behav. Ther. Exp. Psychiatry. 2010; 41(4):331-337. https://doi.org/10.1016/j.jbtep.2010.03.003 PMid:20394916

8. Bryazgunov IP, Mikhailov AN, Stolyarova EV. Posttraumatic stress disorder in children and adolescents. Moscow: PH “Medpraktika-M"; 2008.

9. Bundalo NL. Chronic post-traumatic stress disorder. Krasnoyarsk: KrasSMU; 2009.

10. Christiansen DM, Elklit A. Risk factors predict post- traumatic stress disorder differently in men and women. Ann. Gen. Psychiatry. 2008;7:1-37. https://doi.org/10.1186/1744-859X-7-24 PMid:19017412 PMCid:PMC2603007

11. Delahanty DL. Toward the Predeployment Detection of Risk for PTSD. Am. Journal Psychiatry. 2011;168(1): 9-11. https://doi.org/10.1176/appi.ajp.2010.10101519 PMid:21205813

12. Dmitrieva TB, Drozdov FZ, Kogan BM. Clinical neurochemistry in psychiatry. Moscow:RIO SSC SSP them. V.P. Serbian; 1998.

13. Dohrenwend BP, Neria Y, Turner JB. Positive tertiary appraisals and posttraumatic stress disorder in U. S. male veterans of the war in Vietnam: the roles of positive affirmation, positive reformulation, and defensive denial. Journal Consult. Clin. Psychol. 2005;72(3):417-433. https://doi.org/10.1037/0022-006X.72.3.417 PMid:15279526

14. Elklit A, Reinholt N, Nielsen LH. Posttraumatic stress disorder among bereaved relatives of cancer patients. Journal Psychosoc. Oncol. 2010;28(4):399-412. https://doi.org/10.1080/07347332.2010.488142 PMid:20623415

15. Feldner MT, Monson CM, Friedman MJ. Critical Analysis of Approaches to Targeted PTSD Prevention: Current Status and Theoretically Derived Future Directions. Behav. Modif. 2007;31(1):80-116. https://doi.org/10.1177/0145445506295057 PMid:17179532

16. Gordon JG, Asmundson PD. PTSD Diagnostic Criteria: Understanding Etiology and Treatment. Am. Journal Psychiatry. 2009;166(6):726-732. https://doi.org/10.1176/appi.ajp.2009.08121799 PMid:19487404

17. Horowitz MJ. Stress response syndromes and their treatment. V.Hamilton \& D.Warburton (Eds.). Human stress and cognition. New York: Wiley; 1980.

18. Jind L, Elklit A, Christiansen D. Cognitive schemata and processing among parents bereaved by infant death. Journal Clin. Psychol. Med. Settings. 2010;17(4):366-377. https://doi.org/10.1007/s10880-010-9216-1 PMid:21110073

19. Kaplan GI, Sadok BJ. Clinical Psychiatry: Moscow; 1994.

20. Kitaev-Smyk LA. Post-traumatic stress disorder - are they due to an unsatisfied thirst for revenge, or an unquenchable thirst for love? Questions of psychology of extreme situations. 2008;2:2-16.

21. Krasnov VN. Affective spectrum disorders. Moscow: Practical medicine; 2011. 
22. Krent D, Melhem N, Donohoe B, Walker M. The Incidence and Course of Depression in Bereaved Youth 21 Months After the Loss of a Parent to Suicide, Accident, or Sudden Natural Death. Am. Journal Psychiatry. 2009;166:786-794.

23. Kuo JR, Linehan MM. Disentangling emotion processes in borderline personality disorder: physiological and selfreported assessment of biological vulnerability, baseline intensity, and reactivity to emotionally evocative stimuli. Journal Abnorm. Psychol. 2009;118:531-544. https://doi.org/10.1037/a0016392 PMid:19685950 PMCid:PMC4277483

24. Litvintsev SV, Snedkov AM, Reznik EV. Combat mental injury: a guide for doctors. Moscow: Medicine; 2005.

25. Möller-Leamküller AM. Stress in society and stress-related disorders in terms of gender differences. Social and Clinical Psychiatry. 2004;4:5-11.

26. Narov MYu, Semke VYa, Aksenov MM. Early diagnosis and psychotherapeutic correction of PTSD as prevention of comorbid alcohol addiction in combatants. Siberian Journal of Psychiatry and Narcology. 2007;1(44):69-71.

27. Nechiporenko VV, Shamrey VK. Suicidology: issues of the clinic, diagnosis and prevention. St. Petersburg: Military Medical Academy; 2007.

28. Nemeroff CB, Bremner JD, Foa EB. Posttraumatic stress disorder: A state-of-the-science review. Journal of Psychiatric Research. 2006;40:1-21. https://doi.org/10.1016/j.jpsychires.2005.07.005 PMid:16242154

29. Nikityuk DB, Miroshkin DV. Immune structures with increased physical exertion. Morphology. 2008;133(2):85-90.

30. Nikityuk DB, Miroshkin DV, Bukavneva NS. Clinical and anthropological approaches. Morphological statements. 2008;1(2):56-62.

31. Reznik AM. Subjective assessment by the military of conditions and methods for reducing combat stress. Journal of Neurologists and Psychiatrist. 2009;12:38-40.

32. Shcherbakova OI. Formation managers stress management skills. Modern training and coaching: new opportunities in business and education. Moscow:Russian University of Economics; 2017.

33. Shcherbakova OI, Chernykh AK, Balahanova DK, Midova VO. Stress control practices for mental balance improvement in academic sports. Teoriya i Praktika Fizicheskoy Kultury. 2018;9(1):22-23.

34. Snedkov EV. The problem of nosological independence post-traumatic stress disorder. Journal of Neurology and Psychiatry. 2009;12:8-11.

35. Sukiasyan SG. On some aspects of the dynamics of post-traumatic stress disorders in combatants. Social and Clinical Psychiatry. 2009;1:12-18.

36. Tadevosyan A. Stressology as a theoretical concept of stress disorders (analytical review). Russian Psychiatric Journal. 2006;6:86-92.

37. Tarabrina NV. Workshop on the psychology of post-traumatic stress. St. Petersburg: Peter; 2009.

38. Tsygankov BD, Dzhangildin YuT, Schetinina EA. Psycho-psychotherapeutic correction of mental disorders resulting from stress. Siberian Journal of Psychiatry and Narcology. 2006;4(1):301-302.

39. Voloshin VM. (2004). Typology of chronic post-traumatic stress disorder. Journal of Neurology and Psychiatry. 2004; $1: 17-23$.

40. Wasserman LI, Ababkov VA, Trifonova EA. Coping with stress: theory and psychodiagnostics. St. Petersburg: Speech; 2010.

41. Wilcox HC, Storr CL, Breslau N. Posttraumatic Stress Disorder and Suicide Attempts in a Community Sample of Urban American Young Adults. Arch. Gen. Psychiatry. 2009;66(3):305-311. https://doi.org/10.1001/archgenpsychiatry.2008.557 PMid:19255380 PMCid:PMC6003890 